\title{
To Zika and destroy: an antimalarial drug protects fetuses from Zika infection
}

\author{
Bin Cao ${ }^{1,2}$, Meghal N Sheth ${ }^{1,2}$ \& Indira U Mysorekar*,1,2,3 \\ ${ }^{1}$ Department of Obstetrics \& Gynecology, Washington University School of Medicine, 660 South Euclid Avenue, St Louis, MO \\ 63110, USA \\ ${ }^{2}$ Center for Reproductive Health Sciences, Washington University School of Medicine, 660 South Euclid Avenue, St Louis, MO \\ 63110, USA \\ ${ }^{3}$ Department of Pathology \& Immunology, Washington University School of Medicine, 660 South Euclid Avenue, St Louis, MO \\ 63110, USA \\ * Author for correspondence: Tel.: +1 314747 1329; Fax: +1 314747 1350; imysorekar@wustl.edu
}

\begin{abstract}
"Given that HCQ is relatively inexpensive and readily available globally, including in low- and middle-income countries, HCQ may prove to be an ideal therapeutic to treat pregnant women who test positive for ZIKV."”
\end{abstract}

First draft submitted: 25 September 2017; Accepted for publication: 10 October 2017; Published online: 5 January 2018

Keywords: autophagy $\bullet$ hydroxychloroquine $\bullet$ placenta $\bullet$ pregnancy

\section{Zika virus \& human pregnancy}

Zika virus (ZIKV) is a member of the Flaviviridae family of RNA viruses and is closely related to other flaviviruses such as dengue virus, West Nile virus and yellow fever virus [1]. ZIKV was first isolated from a Rhesus macaque in the Zika forest in Uganda in 1947 [2] but, until recently, it was not considered a significant pathogen because it caused a self-limiting illness characterized by mild fever, headache, arthralgia, conjunctivitis and rash. However, during recent epidemics, ZIKV has been associated with devastating neurological sequelae in adults, such as Guillain-Barré syndrome. Furthermore, ZIKV infection in pregnant women can induce fetal complications, such as microcephaly, intrauterine growth restriction and fetal demise [3]. The rapid spread of ZIKV across the Americas and the virus' serious threat to fetal development propelled WHO to declare the ZIKV epidemic an international public health emergency in early 2016. Given the lack of antiviral therapies that are effective against ZIKV, we urgently need to develop therapies to prevent ZIKV-induced fetal defects in infected pregnant women.

\section{Animal models of ZIKV infection in pregnancy}

In preclinical work aimed at developing such therapies, researchers have developed several mouse models of ZIKV infection, many of which require overcoming the natural murine immune resistance to ZIKV [4]. In the model our research team has used pregnant mice lacking an interferon receptor subcutaneously injected with ZIKV. In this model, ZIKV entered the placenta, replicated within feto-placental trophoblasts and fetal endothelial cells and caused extensive placental damage including reduced placental size and increased numbers of apoptotic trophoblasts and nucleated fetal erythrocytes [5]. Moreover, ZIKV was able to cross the placenta, which normally serves as a formidable barrier to pathogens, to replicate within the brain and impair fetal brain development [5-7].

\section{Autophagy \& the placental barrier}

In seeking a therapeutic that could prevent ZIKV placental infection, we drew on previous studies showing that autophagy, a cellular pathway that targets intracellular components for lysosomal degradation, is a critical component of the placental barrier against pathogens [8]. Additionally, ZIKV infection induces autophagic flux in human skin cells, suggesting that autophagy plays a role in ZIKV pathogenesis [9]. We first confirmed that ZIKV also induces autophagy in human trophoblasts in vitro and in mouse placentas in vivo [10]. Next, we showed that upon ZIKV infection, pregnant mice deficient for a key autophagy gene, Atg16L1 (Atg16l1 ${ }^{H M}$ ), had significantly lower ZIKV titers in both the placentas and fetal brains than the wild-type mice. Moreover, pups from ZIKV-infected

Future $\because$ Medicine 
Atg16l1 ${ }^{H M}$ mice were larger than those from ZIKV-infected wild-type mice, and placentas from Atg16l1 ${ }^{H M}$ mice were significantly less damaged by ZIKV infection than placentas from wild-type mice. However, $A \operatorname{tg} 16 l 1^{H M}$ mice had similar maternal serum and brain ZIKV titers as wild-type mice. Further experiments confirmed that deletion of Atg16l1 exclusively in trophoblasts was sufficient to limit ZIKV maternal-fetal transmission. However, deletion of Atg16l1 exclusively in trophoblasts did not reduce ZIKV titers in fetal heads, supporting a previous report that autophagy plays a role in ZIKV infection of the fetal neurons [11]. Together, these findings suggest that autophagy promotes ZIKV infection in the placenta.

\section{A new use for an old drug: hydroxychloroquine limits ZIKV infections in pregnancy by inhibiting autophagy}

Given that loss of a key autophagy gene reduced placental ZIKV transmission, we tested the autophagy inhibitors bafilomycin A1, 3-methyladenine and chloroquine and found that all three were able to reduce ZIKV titers in human trophoblasts in vitro [10]. Hydroxychloroquine (HCQ), the hydroxyl derivative of chloroquine, was an attractive candidate drug for in vivo tests in mice for several reasons. First, like chloroquine, HCQ inhibits autophagy by blocking autophagosome-lysosome fusion and lysosomal degradation [12]. Second, HCQ is an antimalarial drug that has also been used for years to treat chronic autoimmune disorders, particularly systemic lupus erythematosus, in pregnant women [13]. Finally, HCQ has antiviral effects against flaviviruses, HIV and SARSV [14,15].

We administered HCQ to ZIKV-infected mice and found that it greatly reduced ZIKV titers in placentas and fetal heads. Moreover, ZIKV-induced placental damage was prevented by HCQ treatment, and pups from HCQ-treated ZIKV-infected mice were larger than those from untreated ZIKV-infected mice [10]. Finally, our data suggested that the anti-ZIKV effects of HCQ depend on HCQ's ability to inhibit autophagy, as placental and fetal viral titers were equivalent between $\operatorname{Atg} 1611^{\mathrm{HM}}$ mice treated with and without HCQ [10].

Our work provides a new perspective in understanding the mechanism of HCQ's anti-ZIKV effects during pregnancy. Recent studies have shown that ZIKV nonstructural proteins block the Akt-mTOR pathway in human fetal neural stem cells, which may contribute to autophagy activation as mTOR negatively regulates autophagy [11]. Future work untangling the ZIKV-autophagy interaction will help elucidate the molecular mechanism underlying why autophagy inhibition blocks ZIKV infection and maternal-fetal transmission. Although the mechanisms by which loss or inhibition of autophagy reduces ZIKV infection are currently unknown, our human in vitro and mouse in vivo data indicate that HCQ is a promising therapeutic candidate to prevent adverse pregnancy outcomes in ZIKV-infected women.

Taking HCQ treatment from the bench to the bedside to treat pregnant ZIKV-infected women To be used clinically to combat ZIKV infections in pregnancy, HCQ must be demonstrated to be safe for use during pregnancy. Several studies suggest that it is. For example, in a retrospective study of pregnant patients with chronic rheumatologic conditions, 133 women taking HCQ were compared with a control group of 53 pregnant women not taking HCQ. No significant differences in outcomes, such as live birth rate and infant vision, hearing, growth or development, were seen between the two groups [16]. In a prospective follow-up study of women taking HCQ during pregnancy to treat rheumatologic disease, no significant congenital malformations or vision or neurodevelopmental defects were observed [17]. Finally, a systematic review and meta-analysis examined the effects of HCQ on pregnant women with autoimmune diseases and concluded that HCQ did not appear to increase the risk of adverse pregnancy outcomes [13]. Given these studies, HCQ continues to be recommended by the American Congress of Obstetricians and Gynecologists for treatment of lupus and other rheumatological disorders throughout pregnancy. Given that HCQ is relatively inexpensive and readily available globally, including in low- and middle-income countries, HCQ may prove to be an ideal therapeutic to treat pregnant women who test positive for ZIKV.

\section{Acknowledgements}

The authors thank D Frank and B Liang for comments on the manuscript. 
organization or entity with a financial interest in or financial conflict with the subject matter or materials discussed in the manuscript apart from those disclosed.

No writing assistance was utilized in the production of this manuscript.

\section{References}

1. Lazear HM, Diamond MS. Zika virus: new clinical syndromes and its emergence in the western hemisphere. J. Virol. 90(10), 4864-4875 (2016).

2. Plourde AR, Bloch EM. A literature review of Zika virus. Emerg. Infect. Dis. 22(7), 1185-1192 (2016).

3. Chibueze EC, Tirado V, Lopes $\mathrm{KD}$ et al. Zika virus infection in pregnancy: a systematic review of disease course and complications. Reprod. Health 14(1), 28 (2017).

4. Morrison TE, Diamond MS. Animal models of Zika virus infection, pathogenesis and immunity. J. Virol. 91(8), pii: e00009-17 (2017) (Epub ahead of print).

5. Miner JJ, Cao B, Govero J et al. Zika virus infection during pregnancy in mice causes placental damage and fetal demise. Cell 165(5), 1081-1091 (2016).

6. Cao B, Diamond MS, Mysorekar IU. Maternal-fetal transmission of Zika virus: routes and signals for infection. J. Interferon Cytokine Res. 37(7), 287-294 (2017).

7. Mysorekar IU, Diamond MS. Modeling Zika virus infection in pregnancy. N. Engl. J. Med. 375(5), 481-484 (2016).

8. Cao B, Macones C, Mysorekar IU. ATG16L1 governs placental infection risk and preterm birth in mice and women. JCI Insight 1(21), e86654 (2016).

9. Hamel R, Dejarnac O, Wichit S et al. Biology of Zika virus infection in human skin cells. J. Virol. 89(17), 8880-8896 (2015).

10. Cao B, Parnell LA, Diamond MS, Mysorekar IU. Inhibition of autophagy limits vertical transmission of Zika virus in pregnant mice. J. Exp. Med. 214(8), 2303-2313 (2017).

11. Liang Q, Luo Z, Zeng J et al. Zika virus NS4A and NS4B proteins deregulate Akt-mTOR Signaling in human fetal neural stem cells to inhibit neurogenesis and induce autophagy. Cell Stem Cell 19(5), 663-671 (2016).

12. Rubinsztein DC, Codogno P, Levine B. Autophagy modulation as a potential therapeutic target for diverse diseases. Nat. Rev. Drug Discov. 11(9), 709-730 (2012).

13. Kaplan YC, Ozsarfati J, Nickel C, Koren G. Reproductive outcomes following hydroxychloroquine use for autoimmune diseases: a systematic review and meta-analysis. Br. J. Clin. Pharmacol. 81(5), 835-848 (2016).

14. Savarino A, Di Trani L, Donatelli I, Cauda R, Cassone A. New insights into the antiviral effects of chloroquine. Lancet Infect. Dis. 6(2), 67-69 (2006).

15. Wang LF, Lin YS, Huang NC et al. Hydroxychloroquine-inhibited dengue virus is associated with host defense machinery. J. Interferon Cytokine Res. 35(3), 143-156 (2015).

16. Costedoat-Chalumeau N, Amoura Z, Duhaut P et al. Safety of hydroxychloroquine in pregnant patients with connective tissue diseases: a study of one hundred thirty-three cases compared with a control group. Arthritis Rheum. 48(11), 3207-3211 (2003).

17. Motta M, Tincani A, Faden D et al. Follow-up of infants exposed to hydroxychloroquine given to mothers during pregnancy and lactation. J. Perinatol. 25(2), 86-89 (2005). 
